\title{
Mehr Mitbestimmung in Europa - Mitbestimmung in Deutschland sichern
}

\author{
NORBERT KLUGE
}

\begin{abstract}
„Mitbestimmung“ hat in letzter Zeit Punkte in der öffentlichen Wahrnehmung gesammelt - nicht zuletzt, weil sie sich bei der Überwindung der letzten Finanz- und Wirtschaftskrise nicht nur für Arbeitnehmerinnen und Arbeitnehmer als Vorteil erwiesen hat, sondern auch für die Unternehmen und die Gesellschaft. Regierungen aus Ländern mit gesetzlicher Mitbestimmung sind deshalb in der Verantwortung, diesen Vorteil gegen immer noch fortschreitende wirtschaftliche Liberalisierungspolitiken abzusichern. Nur so kann die neue Europäische Kommission motiviert werden, den rechtlichen Rahmen für Unternehmen so zu setzen, dass sich Mitbestimmung als Prinzip für nachhaltige Unternehmensführung mehr und mehr durchsetzen kann.
\end{abstract}

Ein politischer Hebel dafür ist das europäische Gesellschaftsrecht. Hier hat die Europäische Kommission gesetzgeberische Kompetenz. Ihre vertragliche Aufgabe ist es vor allem, die im EU-Binnenmarkt gegebene Niederlassungsfreiheit und Mobilität von Unternehmen zu erleichtern. Einen zweiten Schwerpunkt sieht die europäische Politik darin, die Rechte von Aktionären gegenüber TopManagern zu stärken. Gesetzliche Beteiligungsrechte über Mitbestimmung für Arbeitnehmerinnen und Arbeitnehmer, wie sie in Deutschland, Österreich oder in Schweden und Dänemark existieren, erscheinen in diesem Kontext als nationale Besonderheiten. In der Logik der EU-Kommission sind solche Rechte ein Fremdkörper im europäischen Gesellschaftsrecht. Sie müssen, so diese Perspektive, wie auch immer rechtlich eingepasst oder mühselig überwunden werden.

In Deutschland verfolgen insbesondere die Arbeitgeberverbände seit mehr als zehn Jahren die Agenda, Mitbestimmung „europakonform“ zu machen. Zwei Fliegen könnten mit einer Klappe geschlagen werden:

- Mitbestimmungsrechte würden substanziell in Deutschland geschmälert;

- Mitbestimmung würde auf diesem Weg als Hindernis für ein weitergehendes europäisches Gesellschaftsrecht aus dem Weg geräumt.
Mehr oder weniger ausgesprochen sollen Mitbestimmungsrechte geschleift werden: Beteiligung im Aufsichtsrat auf ein Drittel der Sitze reduzieren, externe gewerkschaftliche Vertreter aus dem Aufsichtsrat entfernen sowie Aufsichtsräte verkleinern, die nach deutschem Mitbestimmungsgesetz in großen Unternehmen mit mehr als 20.000 Beschäftigten insgesamt 20 Mitglieder haben.

Im Zuge dieser Arbeitgeberinitiative, aber auch infolge der einseitig liberalen gesellschaftsrechtlichen Ausrichtung der EU-Kommission, ist die Unternehmensmitbestimmung in Deutschland in den Zangengriff geraten. Sie hat dem Druck bisher im Großen und Ganzen standgehalten, wird allerdings, wie die folgenden Beispiele zeigen, vielfach attackiert:

(1) Die Europäische Aktiengesellschaft (SE) mit ihrer „Vorher-Nachher-Regelung “ hat zwar nicht zur Flucht aus der Mitbestimmung durch deutsche Unternehmen geführt. Dennoch wurde die SE-Umwandlung in vielen Fällen zur Verkleinerung des Aufsichtsrats genutzt. Gerade kleinere und mittelständische Unternehmen nutzen die SE dazu, vor dem Erreichen von Schwellenwerten (ab 501 Beschäftigten Drittelbeteiligung, ab 2001 Beschäftigten $50 \%$ Beteiligung von Arbeitnehmervertretern in Aufsichtsräten) den Ausgangszustand einzufrieren und damit die Anwendung des deutschen Rechts im Vorfeld zu unterlaufen.

(2) Weitere gesellschaftsrechtliche EU-Richtlinien, wie diejenige zur grenzüberschreitenden Fusion von Unternehmen und der damit verbundene Wechsel des Unternehmenssitzes von einem ins andere Land, enthalten Regelungen zur Bewahrung von zuvor bestehender Mitbestimmung. Allerdings beobachten wir, dass der dafür gesetzlich vorgesehene Mechanismus zur Aushandlung einer entsprechenden Beteiligungsvereinbarung oft nicht zur Anwendung kommt. Damit wird bestehende Mitbestimmung faktisch unterlaufen.
(3) Hinzu kommt eine wachsende Anzahl von mittlerweile fast 100 deutschen Unternehmen, die sich der Mitbestimmung durch die Verlegung ihres Firmensitzes in einen anderen EU-Mitgliedstaat entziehen, obwohl ihre geschäftlichen Tätigkeiten weiterhin hauptsächlich in Deutschland stattfinden.

Seit einiger Zeit konzentriert sich die Fortentwicklung des europäischen Gesellschaftsrechts darauf, den Unternehmen zu gestatten, ihren Sitz in einem EU-Mitgliedstaat registrieren zu lassen, die reale Verwaltung des Unternehmens aber in einem anderen EU-Mitgliedsstaat zu lokalisieren. Im Extremfall könnte eine Briefkastenfirma in einem EU-Mitgliedstaat mit besonders günstigen nationalen Regelungen für Unternehmen ausreichen, um die europaweiten Aktivitäten eines Unternehmens einzig nach dem Gesellschaftsrecht in diesem Land zu steuern. Neben den negativen Folgen für Anleger-, Gläubiger-, und Verbraucherschutz ließe sich so dann auch das „Hindernis Mitbestimmung “ im europäischen Gesellschaftsrecht ausräumen. Obwohl gesetzliche Beteiligung von Arbeitnehmerinnen und Arbeitnehmern an der Unternehmensspitze für die gesamte Wirtschaft in zwölf EU-Mitgliedstaaten existiert Deutschland also kein exotischer Ausnahmefall ist -, gibt es eben weitere 16 EU-Mitgliedstaaten, die diesen Schutz von Arbeitnehmerinteressen eben nicht vorsehen. Und nationale Mitbestimmungsmöglichkeiten enden bekanntlich an den Grenzen des jeweiligen Landes. Es besteht also in dieser Hinsicht freie Auswahl für die Unternehmen, via europäisch sanktioniertem Recht „Regimeshopping “ $z u$ betreiben und die günstigsten Bedingungen für sich zu optimieren. Dies gilt natürlich auch für die Frage, in welchem Land Unternehmensgewinne versteuert werden.

Insofern kann jede weitere Liberalisierung im europäischen Gesellschaftsrecht, wie bspw. die Möglichkeit zur Aufsplittung des Unternehmenssitzes, nicht nur als Einladung zur Umgehung von Mitbestimmung gewertet werden, sondern auch als weiterer Steigbügel zur 
Umgehung von nationalen Steuergesetzen innerhalb der EU.

Kurz vor dem - noch abwendbaren - nächsten Etappensieg stehen Aktionäre und Unternehmen (und die mit ihnen verbundenen geschäftlich interessierten Beraterindustrien) mit dem Plan, für Europa die Ein-Personen-Gesellschaft (SUP) zuzulassen. Auf Basis einer europäischen Rechtsgrundlage sollen vorgeblich vor allem Klein- und Mittelunternehmen mühelos und ohne bürokratische Hürden in einem anderen EU-Mitgliedstaat Unternehmen aus dem Nichts gründen und registrieren lassen können. Sie benötigen dafür keinerlei transnationale Geschäftsdimension. Im Ergebnis entsteht eine nationale Gesellschaft, aber ohne Anwendung möglicherweise höherer Standards des nationalen Rechts. Kern der SUP ist der - erneute - Versuch, die Aufsplittung des Unternehmenssitzes europäisch zu regeln und damit der einschlägigen Rechtsprechung des Europäischen Gerichtshofes (EUGH) die höheren Weihen europäischer Gesetzgebung zu geben. Das war auch schon das Anliegen der Europäischen Privatgesellschaft (SPE). Sie war letztlich am Widerstand einiger Regierungen genau an dieser Frage gescheitert und als europäisches Projekt aufgegeben worden.

Obwohl die SUP in den schönen Mantel des vermeintlichen Vorteils für Klein- und Mittelbetriebe gehüllt ist, sind die vorgetragenen Gegenargumente gegen die SUP/EPG noch immer die gleichen: Weniger Rechtssicherheit auf nationaler Ebene durch faktische Aufweichung nationaler Standards, weniger Gläubiger- und Konsumentenschutz, Einladung zur Steueroptimierung, Einladung zur Mitbestimmungsflucht. Hier geht es also offenbar um mehr als das Anliegen für Klein- und Mittelunternehmen. Mit der Absicht des exklusiven Vorteils für Aktionäre und Unternehmen wird versucht, mit dem falschen Etikett der Förderung unternehmerischer Freiheit und Mobilität politische Mehrheiten zu organisieren. Dabei ginge es jetzt darum, Europaverdruss der Bürgerinnen und Bürger und das politische Momentum der Luxleaks Affäre entschieden zu nutzen, um über das europäische Gesellschaftsrecht mehr Rechtssicherheit und Anerkennung ihrer Leistungen für Bürgerinnen und Bürger und Arbeitnehmerinnen und Arbeitnehmer in Europa einzuziehen. Das Unternehmen ist nicht nur eine private Veranstaltung. Es muss sich gesellschaftlich und sozial verantworten. Alle Beteiligten tragen mit ihrer Leistung gleichermaßen zum Erfolg eines Unternehmens bei.

Das muss sich in den rechtlichen Verpflichtungen eines Unternehmens widerspiegeln: Eu- ropäisches Recht muss Standards zur Beteiligung aller Interessengruppen am Unternehmen setzen und sich nicht auf neue, vor allem börsengetriebene, Transparenzpflichten wie extensives Reporting oder die Verpflichtung zu CorporateGovernance-Richtlinien für Investoren und Unternehmenslenker beschränken. Die obligatorische Beteiligung in Aufsichts- und Verwaltungsräten, wie sie in vielen EU-Mitgliedstaaten gute und gelebte Praxis ist, muss elementarer Bestandteil des europäischen Gesellschafts- und Wirtschaftsrechts werden. Mitbestimmung ist ein gleichberechtigtes Element guter Unternehmensführung. Sie ist weder Fremdkörper noch gar ein historisches Relikt.

Nicht eine erneuerte „EU-Aktionärsrichtlinie“, wie sie gegenwärtig auf dem Tisch des Europäischen Parlaments liegt, sondern eine „EUStakeholder-Richtlinie“ gehört eigentlich auf die politische Tagesordnung. In ihr müssten rechtliche Mindeststandards für die Beteiligung aller relevanten Interessengruppen am Unternehmen - Arbeitnehmer, Anteilseigner, Manager, Vertreter der Region und der Unternehmensumwelt für die europäische Binnenmarktrechtsetzung festgelegt sein.

Anstatt wie aktuell ein schon totgerittenes Pferd wie SUP oder EPG weiterzureiten oder mit dem sog. REFIT ${ }^{1}$ das Etikett Bürokratieabbau zum Schleifen von Arbeitnehmerrechten in Europa zu missbrauchen, gehört jetzt ein Vorschlag für eine Europäische Sitzverlegungsrichtlinie auf den Tisch: Sie muss definieren, unter welchen Umständen und unter Beibehaltung welcher Standards Unternehmen ihren Sitz von einem EU-Mitgliedstaat in einen anderen verlegen können, ohne nationales Recht zur Arbeitnehmerbeteiligung, aber auch zur Unternehmensbesteuerung oder zum Gläubiger- und Konsumentenschutz zu unterlaufen. Ziel der europäischen Gewerkschaften muss es sein, auf diesem Wege Mindeststandards für die Unterrichtung, Anhörung und Mitbestimmung in ganz Europa zu installieren.

Bundestagspräsident Norbert Lammert hatte anlässlich der Konferenz der Hans-Böckler-Stiftung für Aufsichtsräte 2014 die Weiterentwicklung der Mitbestimmung hin zu echter Parität ins Spiel gebracht. ${ }^{2}$ Er hatte dafür sozial- und gesell-

1 REFIT= Regulatory Fitness and Performance Programme, vgl. http://ec.europa.eu/smartregulation/refit/index_en.htm.

2 Siehe Videodokumentation seiner Rede unter http://www.boeckler.de/veranstaltung_44769.htm. schaftspolitische Gründe ins Feld geführt: Unternehmen sind heute Gegenstand von Geschäftsmodellen von internationalen Investoren. Sie sind wenig daran interessiert, als Eigner Verantwortung für den Fortbestand von Unternehmen und Perspektiven für Standorte und Beschäftigung zu übernehmen. Wenn das Potenzial der Mitbestimmung zum sozialen Interessenausgleich in der sozialen Marktwirtschaft aber gewahrt bleiben soll, dann muss die Mitbestimmung gesetzlich gestärkt werden. Das gilt umso mehr mit Blick auf Europa. Deshalb wäre die deutsche Bundesregierung gut beraten, jetzt die Schlupflöcher in der nationalen Gesetzgebung zu schließen. Sonst wird die Mitbestimmung auch in Deutschland zum Inselreich und der Vorteil Mitbestimmung für Wirtschaft und Gesellschaft ist verspielt.

\section{AUTOR}

NORBERT KLUGE, Dr., ist Leiter der Abteilung Mitbestimmungsförderung in der Hans-BöcklerStiftung, Düsseldorf. Arbeitsschwerpunkte: Mitbestimmung für das nachhaltige Unternehmen, Fortentwicklung von Konzeption und Praxis der Mitbestimmung, Europäisierung der Mitbestimmung.

norbert-kluge@boeckler.de 